\title{
Position-space approach to hadronic light-by-light scattering in the muon $g-2$ on the lattice
}

\author{
Nils Asmussen*, Jeremy Green, Harvey B. Meyer and Andreas Nyffeler \\ PRISMA Cluster of Excellence, Institut für Kernphysik and Helmholtz Institute Mainz, \\ Johannes Gutenberg-Universität Mainz, 55099 Mainz, Germany \\ E-mail: \{asmussen, green, meyerh, nyffeler\}akph.uni-mainz.de
}

\begin{abstract}
The anomalous magnetic moment of the muon currently exhibits a discrepancy of about three standard deviations between the experimental value and recent Standard Model predictions. The theoretical uncertainty is dominated by the hadronic vacuum polarization and the hadronic lightby-light (HLbL) scattering contributions, where the latter has so far only been fully evaluated using different models. To pave the way for a lattice calculation of HLbL, we present an expression for the HLbL contribution to $g-2$ that involves a multidimensional integral over a position-space QED kernel function in the continuum and a lattice QCD four-point correlator. We describe our semi-analytic calculation of the kernel and test the approach by evaluating the $\pi^{0}$-pole contribution in the continuum.
\end{abstract}

34th annual International Symposium on Lattice Field Theory

24-30 July 2016

University of Southampton, UK

${ }^{*}$ Speaker. 


\section{Introduction}

One of the most precisely measured physical quantities is the anomalous magnetic moment of the muon $a_{\mu}=\frac{g_{\mu}-2}{2}$. The Standard Model provides predictions of similar accuracy as the measurements. Comparing the theoretical and experimental value leads to a very stringent test of the Standard Model. A long-standing discrepancy of three standard deviations or more is observed in $a_{\mu}$,

$$
a_{\mu}= \begin{cases}116592089(63) \times 10^{-11} & \text { experiment }[1,2] \\ 116591790(65) \times 10^{-11} & \text { theory }[3] .\end{cases}
$$

To reduce the uncertainty, experiments planned at Fermilab and at J-PARC [4] aim to improve the experimental uncertainty by a factor of four. To profit most from these efforts, the theoretical uncertainty must be reduced by a similar amount. Despite not being the largest contributions to $a_{\mu}$, the hadronic vacuum polarization (HVP) $\left(\mathscr{O}\left(\alpha^{2}\right)\right)$ and hadronic light-by-light scattering contribution (HLbL) ( $\left.\mathscr{O}\left(\alpha^{3}\right)\right)$ contribute most to the theoretical uncertainty of $a_{\mu}$.

Unlike the HVP, the HLbL is not fully related to any cross section. The estimates of the HLbL rely on models, which leads to large uncertainties. In the phenomenological treatment one tries to reduce model uncertainties for the dominant contributions $\left(\pi^{0}, \eta, \eta^{\prime} ; \pi \pi\right)$ by using experimental input with the help of dispersion relations, see Colangelo et al. [5, 6, 7] and Pauk and Vanderhaeghen [8]. Lattice QCD can provide tests of dispersive approaches [9] and also a direct first-principle estimate of $a_{\mu}^{\mathrm{HLbL}}[10]$.

\section{An expression for $a_{\mu}^{\mathrm{HLbL}}$ adapted to lattice QCD calculations}

The basic idea of our method is to treat the four-point function represented by the blob in Fig. 1 in lattice regularization, while for the remaining (QED) parts, we use continuum, Euclidean position-space perturbation theory in infinite volume $[11,12]$. In this way, we avoid having powerlaw corrections in the volume on $a_{\mu}^{\mathrm{HLbL}}$.

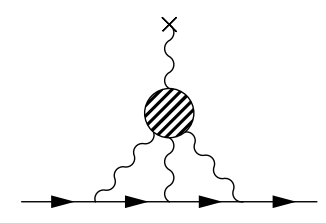

Figure 1: Hadronic light-by-light scattering diagram

In Euclidean space, the matrix element of the electromagnetic current at momentum transfer $k=p^{\prime}-p$ can be decomposed into form factors as

$$
\left\langle\mu^{-}\left(p^{\prime}, s^{\prime}\right)\left|j_{\rho}(0)\right| \mu^{-}(p, s)\right\rangle=-\bar{u}^{s^{\prime}}\left(p^{\prime}\right)\left[\gamma_{\rho} F_{1}\left(k^{2}\right)+\frac{\sigma_{\rho \tau} k_{\tau}}{2 m} F_{2}\left(k^{2}\right)\right] u^{s}(p),
$$

The anomalous magnetic moment is defined as the Pauli form factor at $k^{2}=0, a_{\mu}=F_{2}(0)$. An explicit projection of the vertex to $a_{\mu}$ can be given [13],

$$
F_{2}(0)=\frac{-i}{48 m} \operatorname{Tr}\left\{\left[\gamma_{\rho}, \gamma_{\tau}\right](-i \not p+m) \Gamma_{\rho \tau}(p, p)(-i \not p+m)\right\},
$$


where the HLbL contribution to the vertex reads $\left(\int_{q} \equiv \int \frac{d^{4} q}{(2 \pi)^{4}}, \int_{x} \equiv \int d^{4} x\right)$

$$
\begin{gathered}
\Gamma_{\rho \sigma}\left(p^{\prime}, p\right)=-e^{6} \int_{q_{1}, q_{2}} \frac{1}{q_{1}^{2} q_{2}^{2}\left(q_{1}+q_{2}-k\right)^{2}} \frac{1}{\left(p^{\prime}-q_{1}\right)^{2}+m^{2}} \frac{1}{\left(p^{\prime}-q_{1}-q_{2}\right)^{2}+m^{2}} \\
\left(\gamma_{\mu}\left(i \not p^{\prime}-i q_{1}-m\right) \gamma_{v}\left(i \not p^{\prime}-i q_{1}-i q_{2}-m\right) \gamma_{\lambda}\right) \frac{\partial}{\partial k_{\rho}} \Pi_{\mu \nu \lambda \sigma}\left(q_{1}, q_{2}, k-q_{1}-q_{2}\right), \\
\Pi_{\mu v \lambda \sigma}\left(q_{1}, q_{2}, q_{3}\right)=\int_{x_{1}, x_{2}, x_{3}} e^{-i\left(q_{1} x_{1}+q_{2} x_{2}+q_{3} x_{3}\right)}\left\langle j_{\mu}\left(x_{1}\right) j_{v}\left(x_{2}\right) j_{\lambda}\left(x_{3}\right) j_{\sigma}(0)\right\rangle .
\end{gathered}
$$

We now parametrize the on-shell muon momentum by a unit-vector $\hat{\varepsilon}$ as

$$
p=i m \hat{\varepsilon} \quad\left(p^{2}=-m^{2}\right),
$$

and write the expression in terms of position-space functions,

$$
\begin{aligned}
\Gamma_{\rho \sigma}(p, p) & =-e^{6} \int_{x, y} \widehat{\Pi}_{\rho ; \mu \nu \lambda \sigma}(x, y) K_{\mu \nu \lambda}(x, y, p), \\
K_{\mu \nu \lambda}(x, y, p) & =\gamma_{\mu}\left(i \not p+\not^{(x)}-m\right) \gamma_{v}\left(i \not p+\not^{(x)}+\not^{(y)}-m\right) \gamma_{\lambda} \mathscr{I}(\hat{\varepsilon}, x, y), \\
\mathscr{I}(\hat{\varepsilon}, x, y) & =\int_{q, k} \frac{1}{q^{2} k^{2}(q+k)^{2}} \frac{1}{(p-q)^{2}+m^{2}} \frac{1}{(p-q-k)^{2}+m^{2}} e^{-i(q x+k y)}, \\
\widehat{\Pi}_{\rho ; \mu \nu \lambda \sigma}(x, y) & =\int_{z} i z_{\rho}\left\langle j_{\mu}(x) j_{v}(y) j_{\sigma}(z) j_{\lambda}(0)\right\rangle .
\end{aligned}
$$

Since the scalar function $\mathscr{I}$ is logarithmically infrared divergent, we need to introduce a regulator. However the derivatives in $K_{\mu \nu \lambda}$ remove the divergence, so that the latter tensor is infrared finite.

Our task is now to evaluate the Fourier integral in $\mathscr{I}$. We express it in terms of position-space scalar propagators $G_{m}(x)$,

$$
\begin{aligned}
\mathscr{I}(\hat{\varepsilon}, x, y) & =\int_{u, \mathrm{IR}-\mathrm{reg}} G_{0}(u-y) J(\hat{\varepsilon}, u) J(\hat{\varepsilon}, x-u) \\
J(\hat{\varepsilon}, y) & =\int_{x} G_{0}(x+y) e^{-m \hat{\varepsilon} \cdot x} G_{m}(x) \\
G_{m}(x) & =\frac{m}{4 \pi^{2}|x|} K_{1}(m|x|) \quad\left(K_{1} \text { is a modified Bessel function }\right) .
\end{aligned}
$$

We insert Eq. (2.6) into (2.2), evaluate the trace and obtain an expression of the form

$$
a_{\mu}^{\mathrm{HLbL}}=\hat{F}_{2}(0)=\frac{m e^{6}}{3} \int_{y} \int_{x} \mathscr{L}_{[\rho, \sigma] ; \mu \nu \lambda}(\hat{\varepsilon}, x, y) i \widehat{\Pi}_{\rho ; \mu \nu \lambda \sigma}(x, y) .
$$

We exploit the invariance of $\hat{F}_{2}(0)$ under $\mathrm{O}(4)$ rotations of the muon momentum and average the kernel over the direction of $\hat{\varepsilon}$,

$$
\overline{\mathscr{L}}_{[\rho, \sigma] ; \mu \nu \lambda}(x, y)=\frac{1}{2 \pi^{2}} \int \mathrm{d} \Omega_{\varepsilon} \mathscr{L}_{[\rho, \sigma] ; \mu \nu \lambda}(\hat{\varepsilon}, x, y) \equiv\left\langle\mathscr{L}_{[\rho, \sigma] ; \mu \nu \lambda}(\hat{\varepsilon}, x, y)\right\rangle_{\hat{\varepsilon}} .
$$

Thus we arrive at the master formula [12],

$$
a_{\mu}^{\mathrm{HLbL}}=F_{2}(0)=\frac{m e^{6}}{3} \int_{y} \int_{x} \overline{\mathscr{L}}_{[\rho, \sigma] ; \mu \nu \lambda}(x, y) i \widehat{\Pi}_{\rho ; \mu \nu \lambda \sigma}(x, y) .
$$


The angular average is performed analytically by first expanding the dependence of $J(\hat{\varepsilon}, y)$ on $\hat{\varepsilon} \cdot \hat{y}$ in Chebyshev polynomials of the second kind $U_{n}$ - a special case of the Gegenbauer expansion,

$$
J(\hat{\varepsilon}, y)=\sum_{n \geq 0} z_{n}\left(y^{2}\right) U_{n}(\hat{\varepsilon} \cdot \hat{y}) .
$$

The coefficients $z_{n}$ turn out to be linear combinations of products of two modified Bessel functions. The orthogonality property $\left\langle U_{n}(\hat{\varepsilon} \cdot \hat{x}) U_{m}(\hat{\varepsilon} \cdot \hat{y})\right\rangle_{\hat{\varepsilon}}=\frac{\delta_{n m}}{n+1} U_{n}(\hat{x} \cdot \hat{y})$ can then be exploited.

The formula in Eq. (2.15) shows the QED kernel function $\overline{\mathscr{L}}_{[\rho, \sigma] ; \mu \nu \lambda}$, that weights the positionspace QCD four-point function $\widehat{\Pi}_{\rho ; \mu \nu \lambda \sigma}(x, y)$. The kernel has been averaged over the direction of the muon momentum, so that after contracting the Lorentz indices the integration reduces to a 3-dimensional integration over $x^{2}, y^{2}$ and the angle $\beta$ between $x$ and $y$ (i.e. $x \cdot y=|x||y| \cos \beta$ ),

$$
\int_{y} \rightarrow 2 \pi^{2} \int_{0}^{\infty} \mathrm{d}|y||y|^{3}, \quad \int_{x} \rightarrow 4 \pi \int_{0}^{\infty} \mathrm{d}|x||x|^{3} \int_{0}^{\pi} \mathrm{d} \beta \sin ^{2} \beta .
$$

In the lattice implementation, we plan to make use of the reduction in the integration dimensionality only for the $y$ integral (see section 4). In analytic calculations, the full reduction ought to be exploited. It is also worth noting that the contraction of $\widehat{\Pi}_{\rho ; \mu \nu \lambda \sigma}(x, y)$ with the tensors $\mathscr{G}_{\delta \rho \sigma \mu \alpha \nu \beta \lambda}^{A}$ introduced in Eq. (3.1) projects the QCD four-point function onto a rank-three tensor, which can be decomposed into a smaller number of tensor structures. Finally, the Bose symmetry of the internal vertices,

$$
\widehat{\Pi}_{\rho ; \mu \nu \lambda \sigma}(x, y)=\widehat{\Pi}_{\rho ; v \mu \lambda \sigma}(y, x)=\widehat{\Pi}_{\rho ; \lambda v \mu \sigma}(-x, y-x),
$$

could be used to further symmetrize the kernel $\overline{\mathscr{L}}_{[\rho, \sigma] ; \mu \nu \lambda}(x, y)$.

\section{Semi-analytic calculation of the kernel}

We decompose the QED kernel function $\overline{\mathscr{L}}_{[\rho, \sigma] ; \mu \nu \lambda}(x, y)$ into tensors $T_{\alpha \beta \delta}^{A}(x, y)$ :

$$
\overline{\mathscr{L}}_{[\rho, \sigma] ; \mu \nu \lambda}(x, y)=\sum_{A=I, I I, I I I} \mathscr{G}_{\delta \rho \sigma \mu \alpha \nu \beta \lambda}^{A} T_{\alpha \beta \delta}^{A}(x, y) .
$$

The $\mathscr{G}_{\delta \rho \sigma \mu \alpha \nu \beta \lambda}^{A}$ are sums of products of Kronecker deltas coming from traces of Dirac matrices. The tensors in turn are decomposed into a scalar $S$, a vector $V_{\delta}$ and a tensor part $T_{\beta \delta}$,

$$
\begin{aligned}
& T_{\alpha \beta \delta}^{I}(x, y)=\partial_{\alpha}^{(x)}\left(\partial_{\beta}^{(x)}+\partial_{\beta}^{(y)}\right) V_{\delta}(x, y), \\
& T_{\alpha \beta \delta}^{I I}(x, y)=m \partial_{\alpha}^{(x)}\left(T_{\beta \delta}(x, y)+\frac{1}{4} \delta_{\beta \delta} S(x, y)\right), \\
& T_{\alpha \beta \delta}^{I I I}(x, y)=m\left(\partial_{\beta}^{(x)}+\partial_{\beta}^{(y)}\right)\left(T_{\alpha \delta}(x, y)+\frac{1}{4} \delta_{\alpha \delta} S(x, y)\right) .
\end{aligned}
$$

These parts are given in terms of the function $\mathscr{I}$ by

$$
\begin{array}{rlrl} 
& \text { scalar: } & S(x, y) & =\langle\mathscr{I}\rangle_{\hat{\varepsilon}} \quad(\text { IR regulated }), \\
\text { vector: } & V_{\delta}(x, y) & =\left\langle\hat{\varepsilon}_{\delta} \mathscr{I}\right\rangle_{\hat{\varepsilon}} \\
& \text { tensor: } & T_{\beta \delta}(x, y) & =\left\langle\left(\hat{\varepsilon}_{\delta} \hat{\varepsilon}_{\beta}-\frac{1}{4} \delta_{\delta \beta}\right) \mathscr{I}\right\rangle_{\hat{\varepsilon}} .
\end{array}
$$


This procedure allows us to parametrize the QED kernel by six form factors, each one a function of $x^{2}, y^{2}, x \cdot y$,

$$
\begin{aligned}
S(x, y) & =g^{(0)}, \quad V_{\delta}(x, y)=x_{\delta} g^{(1)}+y_{\delta} g^{(2)} \\
T_{\alpha \beta}(x, y) & =\left(x_{\alpha} x_{\beta}-\frac{x^{2}}{4} \delta_{\alpha \beta}\right) l^{(1)}+\left(y_{\alpha} y_{\beta}-\frac{y^{2}}{4} \delta_{\alpha \beta}\right) l^{(2)}+\left(x_{\alpha} y_{\beta}+y_{\alpha} x_{\beta}-\frac{x \cdot y}{2} \delta_{\alpha \beta}\right) l^{(3)} .
\end{aligned}
$$

We have computed all six form factors and stored their values on a $(|x|,|y|, \cos \beta)$ grid. In that way, the kernel $\overline{\mathscr{L}}_{[\rho, \sigma] ; \mu \nu \lambda}$ can be obtained from the form factors by a cheap computation based on Eqs. (3.1), (3.2) and (3.4). We achieved a precision on the form factors of about five digits using Gaussian quadrature methods. For instance, the vector form factor $g^{(2)}$ is given by

$$
\begin{gathered}
g^{(2)}\left(x^{2}, x \cdot y, y^{2}\right)=\frac{1}{8 \pi y^{2}|x| \sin ^{3} \beta} \int_{0}^{\infty} d u u^{2} \int_{0}^{\pi} d \phi_{1}\left\{2 \sin \beta+\left(\frac{y^{2}+u^{2}}{2|u||y|}-\cos \beta \cos \phi_{1}\right) \frac{\log \chi}{\sin \phi_{1}}\right\} \\
\sum_{n=0}^{\infty}\left\{z_{n}(|u|) z_{n+1}(|x-u|)\left[|x-u| \cos \phi_{1} \frac{U_{n}}{n+1}+\left(|u| \cos \phi_{1}-|x|\right) \frac{U_{n+1}}{n+2}\right]\right. \\
\left.+z_{n+1}(|u|) z_{n}(|x-u|)\left[\left(|u| \cos \phi_{1}-|x|\right) \frac{U_{n}}{n+1}+|x-u| \cos \phi_{1} \frac{U_{n+1}}{n+2}\right]\right\}
\end{gathered}
$$

where $|x-u|=\left(|x|^{2}+|u|^{2}-2|x||u| \cos \phi_{1}\right)^{1 / 2}$ and

$$
\chi=\frac{y^{2}+u^{2}-2|u||y| \cos \left(\beta-\phi_{1}\right)}{y^{2}+u^{2}-2|u||y| \cos \left(\beta+\phi_{1}\right)}, \quad U_{n}=U_{n}\left(\frac{|x| \cos \phi_{1}-|u|}{|u-x|}\right) .
$$

As can be seen in the left panel of Fig. 2, the form factor $g^{(2)}$ is a smooth function of its arguments.

\section{Numerical test of the kernel and aspects of the lattice implementation}

In order to verify the correctness of the QED kernel, we calculated the pion-pole contribution to the four-point function $\widehat{\Pi}_{\rho ; \mu v \lambda \sigma}(x, y)$ using the VMD model (as defined in [14]) for the pion transition form factor. We then computed $a_{\mu}^{\mathrm{HLbL}, \pi^{0}}$ using Eq. (2.15) and compared with the result obtained via momentum-space integration [14]. Fig. 2 (right panel) shows a comparison for two different pion masses. Although the integrals over $|x|$ and $|y|$ are expected to be exponentially convergent at large distances, numerically the convergence is only achieved for $|y|^{\max } \geq 2-3 \mathrm{fm}$ even though the pion mass $m_{\pi}=600-900 \mathrm{MeV}$ is quite large. The cutoff for the $x$ integration was fixed at $|x|^{\max }=4.05 \mathrm{fm}$.

In a lattice QCD implementation, the cost of computing the diagrams with fully connected quark lines can be estimated as follows. For fixed $y$, the $\mathrm{d}^{4} x$ integral is evaluated as the sum $a^{4} \sum_{x}$ over all lattice sites with the help of sequential propagators. If the remaining one-dimensional integral over $|y|$ is done with $N$ evaluations of the integrand, we need $(1+N)$ forward propagators and $6(1+N)$ sequential propagators. We expect to require $N \approx 20$ for a reliable evaluation of the $|y|$ integral. 

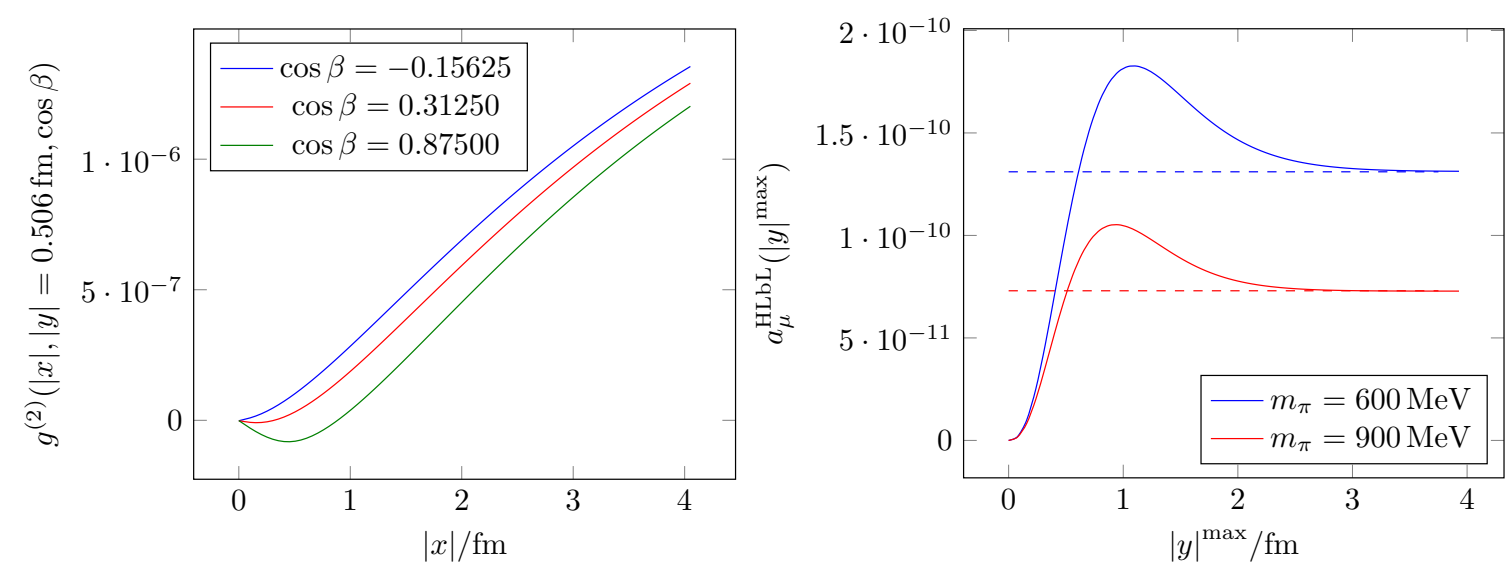

Figure 2: Left: Form factor $g^{(2)}$ for fixed $|y|$ and three different values of $\cos \beta$. Right: Numerical test of the kernel $\overline{\mathscr{L}}_{[\rho, \sigma] ; \mu \nu \lambda}(x, y)$, computing the pion-pole contribution to $a_{\mu}^{\mathrm{HLbL}}$ for two different pion masses, with an upper bound $|y|{ }^{\max }$ for the integral over $|y|$. The dashed lines represent the value computed with momentum-space methods [14].

\section{Summary and outlook}

We presented an explicit formula for $a_{\mu}^{\mathrm{HLbL}}$, Eq. (2.15), consisting of a QED kernel function weighting the Euclidean position-space QCD four-point function [11, 12]. We have completed the calculation of the QED kernel in the continuum in infinite volume. This computational strategy avoids power-law finite-size effects. We exploited the invariance under rotations of the direction of the muon momentum $\hat{\varepsilon}$, by averaging $a_{\mu}^{\mathrm{HLbL}}$ over $\hat{\varepsilon}$. As a result, only the one-dimensional $|y|$ integral must be sampled stochastically. We computed the form factors parametrizing the kernel and stored them on disk, so that the kernel can be computed in a negligible amount of time during the lattice simulation. The correctness of the position-space approach has been tested by computing the pion-pole contribution to $\widehat{\Pi}_{\rho ; \mu \nu \lambda \sigma}(x, y)$ with a VMD form factor and reproducing known results on $a_{\mu}^{\mathrm{HLbL}, \pi^{0}}$ by performing the integrals over $x$ and $y$. This type of calculation may also be used in the future to correct for the leading finite-size effect on $a_{\mu}^{\mathrm{HLbL}}$ in our approach, if the pion transition form factor is computed along the lines of [15].

We are in the process of testing the method on the lattice using non-interacting quarks. It is interesting to compare our approach with the methods presented in [16]. In the latter publication, the most accurate results (for the fully connected contribution) were obtained by effectively using position-space perturbation theory. There, rather than $y$, it is the integral over the difference of the positions of two quark-photon vertices, $r=x-y$, which needs to be performed. Due to the use of a specific muon frame, that integral is four-dimensional. Also, the kernel is fully recomputed for every value of $r$, which may cost a non-negligible fraction of the computing time. While in [16], the kernel was computed on the same space-time lattice as the QCD four-point function, at this conference L. Jin presented a study where the kernel is computed on a larger volume, in order to reduce the power-law corrections in the volume. There are thus a number of similarities, but also significant differences between the methods of the two groups. It is in any case encouraging that Blum et al. [16] obtained a good signal for $a_{\mu}^{\mathrm{HLbL}}$. 
We thank G. von Hippel and H. Wittig for helpful discussions. This work is supported in part by DFG through CRC 1044 "The low-energy frontier of the Standard Model”.

\section{References}

[1] Particle Data Group collaboration, K. A. Olive et al., Review of Particle Physics, Chin. Phys. C38 (2014) 090001.

[2] Muon G-2 collaboration, G. W. Bennett et al., Final Report of the Muon E821 Anomalous Magnetic Moment Measurement at BNL, Phys. Rev. D73 (2006) 072003, [hep-ex/ 0602035 ].

[3] F. Jegerlehner and A. Nyffeler, The Muon g-2, Phys. Rept. 477 (2009) 1-110, [0 902 . 3360 ].

[4] D. W. Hertzog, Next Generation Muon g - 2 Experiments, EPJ Web Conf. 118 (2016) 01015, [1512.00928].

[5] G. Colangelo, M. Hoferichter, M. Procura and P. Stoffer, Dispersive approach to hadronic light-by-light scattering, JHEP 09 (2014) 091, [1402 . 7081].

[6] G. Colangelo, M. Hoferichter, B. Kubis, M. Procura and P. Stoffer, Towards a data-driven analysis of hadronic light-by-light scattering, Phys. Lett. B738 (2014) 6-12, [1408.2517].

[7] G. Colangelo, M. Hoferichter, M. Procura and P. Stoffer, Dispersion relation for hadronic light-by-light scattering: theoretical foundations, JHEP 09 (2015) 074, [1506. 0138 6].

[8] V. Pauk and M. Vanderhaeghen, Anomalous magnetic moment of the muon in a dispersive approach, Phys. Rev. D90 (2014) 113012, [1409.0819].

[9] J. Green, O. Gryniuk, G. von Hippel, H. B. Meyer and V. Pascalutsa, Lattice QCD calculation of hadronic light-by-light scattering, Phys. Rev. Lett. 115 (2015) 222003, [1507 . 01577].

[10] T. Blum, S. Chowdhury, M. Hayakawa and T. Izubuchi, Hadronic light-by-light scattering contribution to the muon anomalous magnetic moment from lattice QCD, Phys. Rev. Lett. 114 (2015) 012001, [1407.2923] and references therein.

[11] N. Asmussen, J. Green, V. Gülpers, G. von Hippel, H.B. Meyer, A. Nyffeler and H. Wittig, Hadronic Light-by-Light Contribution to the Muon Anomalous Magnetic Moment on the Lattice, talk by N. Asmussen at the Conference of the Deutsche Physikalische Gesellschaft, Heidelberg, 23-27 March 2015.

[12] J. Green, N. Asmussen, O. Gryniuk, G. von Hippel, H. B. Meyer, A. Nyffeler et al., Direct calculation of hadronic light-by-light scattering, PoS LATTICE2015 (2016) 109, [1510 . 08384 ].

[13] J. Aldins, T. Kinoshita, S. J. Brodsky and A. J. Dufner, Photon - Photon Scattering Contribution To The Sixth Order Magnetic Moments Of The Muon And Electron, Phys. Rev. D1 (1970) 2378.

[14] M. Knecht and A. Nyffeler, Hadronic light by light corrections to the muon g-2: The Pion pole contribution, Phys. Rev. D65 (2002) 073034, [hep-ph/0111058].

[15] A. Gérardin, H. B. Meyer and A. Nyffeler, Lattice calculation of the pion transition form factor $\pi^{0} \rightarrow \gamma^{*} \gamma^{*},[1607.08174]$.

[16] T. Blum, N. Christ, M. Hayakawa, T. Izubuchi, L. Jin and C. Lehner, Lattice Calculation of Hadronic Light-by-Light Contribution to the Muon Anomalous Magnetic Moment, Phys. Rev. D93 (2016) 014503, [1510.07100]. 